\title{
Chest pain in a young girl
}

\author{
JM Ahmed, MY Salame, GDG Oakley
}

A 28-year-old previously healthy woman presented with left-sided pleuritic chest pain of 18 hours duration. The pain started suddenly in the early hours of the morning while she was at a night club. Initially the pain was dull but several hours later it became more severe and was made worse by deep inspiration. Four hours before the pain started she had taken one 'Ecstasy' (methylenedioxymethamphetamine) and one 'Speed' (methylamphetamine hydrochloride) tablet. There was no history of trauma or asthma and she did not complain of any cough, sputum or haemoptysis. She was on the oral contraceptive pill and never smoked.

On examination she was apyrexial and rather anxious. Her pulse rate was 82 beats $/ \mathrm{min}$ and blood pressure was $116 / 80 \mathrm{mmHg}$. Auscultation of the heart revealed a crunching systolic sound and the chest was clear. Examination of the neck revealed surgical emphysema.

Full blood count, erythrocyte sedimentation rate, blood chemistry, arterial blood gases, electrocardiogram and a water-soluble contrast swallow were all normal. The chest X-rays are shown below (figures 1 and 2).

The patient was given analgesia and she was closely monitored. The chest pain subsided after four days when she was discharged home. A repeat chest X-ray was performed at two and four weeks.

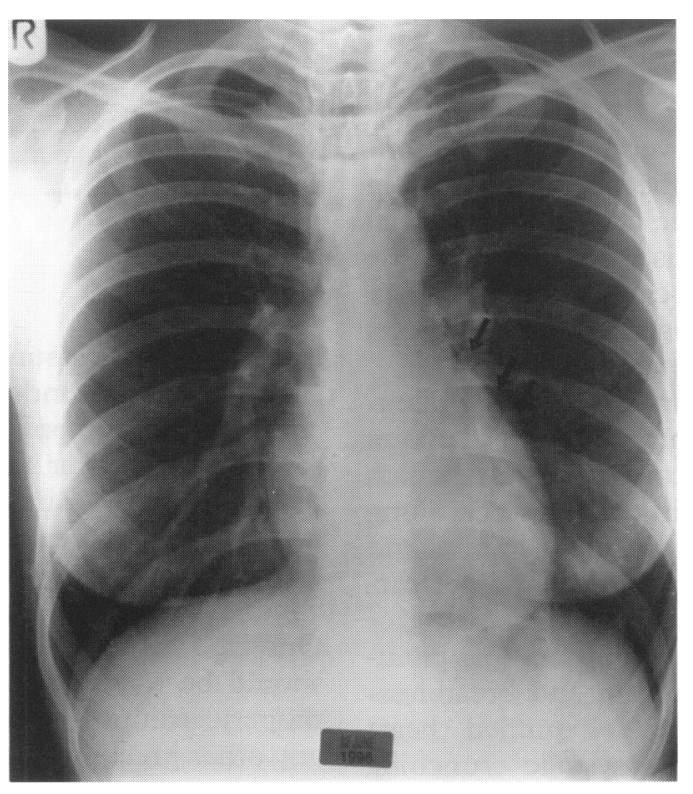

Figure 1 Postero-anterior chest X-ray

Department of Cardiology, Northern General Hospital, Sheffield S5 7AU, UK JM Ahmed

MY Salame

GDG Oakley

Correspondence to Dr GDG Oakley, Consultant

Cardiologist, Department of Cardiology, Northern General Hospital, Herries Road, Sheffield S5 7AU,UK

Accepted 23 July 1997

\section{Questions}

1 What do the postero-anterior and lateral chest X-rays show ?

2 How would you treat this condition? 


\section{Answers}

QUESTION 1

The postero-anterior chest X-ray (figure 1) shows air along the left border of the heart and the lateral chest X-ray (figure 2) shows air in the mediastinum consistent with the diagnosis of pneumopericardium and pneumomediastinum. Pneumopericardium is a very rare disorder resulting from the collection of gas or air in the pericardial sac. The common causes are listed in box 1. Spontaneous pneumopericardium is extremely rare. Serial chest X-rays are required to monitor the progress. In our patient, repeat chest X-rays showed partial absorption of the air at two weeks and complete absorption at four weeks.

QUESTION 2

Spontaneous pneumopericardium is usually a self-limiting condition. Treatment includes observation, analgesics, oxygen, sedation, and careful monitoring. Frequently the air is absorbed spontaneously but rarely, if a large quantity of air is present or the air is under tension, cardiac tamponade may develop. Tension pneumopericardium should be treated with pericardiocentesis and rapid fluid replacement. If hypotension persists, the patient should undergo an urgent pericardiotomy.

\section{Discussion}

Pneumopericardium is a very uncommon condition. It was first described by Bricketeau in 1844. ${ }^{1} \mathrm{He}$ also named the classical pericardial sound associated with this condition 'Bruit de moulin'. The common causes in adults are listed in box 1 . In infants the most common cause is positive pressure ventilation.

The underlying mechanism causing a pneumopericardium is probably related to a sudden rise in alveolar pressure causing rupture which results in the leakage of air which dissects along the perivascular spaces to the mediastinum and enters the pericardial sac through the pericardial reflection of the pulmonary vessels.

The most common symptoms of pneumopericardium are chest pain and breathlessness. Physical signs include muffled heart sounds, bradycardia and a systolic crunching sound called 'Bruit de moulin'. The diagnosis is usually apparent from the chest X-ray and it can be confirmed by CT scan which may also reveal any underlying pathology. Oesophageal lesions are excluded by water-soluble contrast swallow.

In our case report the underlying mechanism could have been due to spontaneous rupture of a congenital pleuropericardial connection, alveolar rupture caused by an increase in

1 Bricketeau M. Observation d'hydropneumopericarde accompagne d'un bruit de fluctuation perceptible a l'oreille. compagne d'un bruit de fluctur

2 Westaby S. Pneumopericardium and tension pneumopericardium after closed chest injury. Thorax 1977;32:91-2.

3 Katzir D, Klinovsly E, Kent V, et al. Spontaneous pneumopericardium: case report and review of the literature. Cardiology 1989;76:305-8.

4 Vennos $\mathrm{AD}$, Templeton PA. Pneumopericardium secondary to esophageal carcinoma. Radiology 1992;182:131-2.

5 Breatnach E, Han SY. Pneumopericardium occurring as a complication of achalasia. Chest 1986;90:292-3.

\section{Pneumopericardium: common causes}

- trauma, blunt and penetrating ${ }^{2}$

- fistula formation between the pericardium and adjacent air-containing organs, eg, bronchus and oesophagus. Examples include bronchogenic carcinoma, ${ }^{3}$ oesophageal and gastric ulcers, oesophageal and gastric carcinoma, ${ }^{4}$ and achalasia $^{5}$

- iatrogenic: thoracocentesis, post-sternal bone marrow aspiration and endotracheal intubation ${ }^{6}$

- spontaneous gas production from gas-producing organisms in the pericardial $\mathrm{sac}^{7}$

- other causes, including pulmonary barotrauma, severe cough, asthma, ${ }^{8}$ cocaine inhalation chlorine gas exposure, emesis, postpartum and, rarely, in athletes

Box 1

\section{Learning points}

- spontaneous pneumopericardium and pneumomediastinum is almost always a self-limiting condition and in most cases air would be eventually absorbed. Management is usually conservative; administration of oxygen, serial chest $\mathrm{X}$-rays and observation are adequate in most cases. In pneumopericardium without tension no intervention is required and most patients would recover within 1-2 weeks

- tension pneumopericardium should be treated with emergency pericardiocentesis

- a careful drug history should be obtained when spontaneous pneumopericardium is present in a young adult without any other obvious cause

Box 2

intra-alveolar pressure due to exertion while she was dancing under the influence of stimulant drugs, or to application of positive ventilatory pressure after taking the drugs. This is usually done by direct mouth-to-mouth contact or through a cardboard cylinder by which the partner applies positive ventilatory pressure which is said to enhance the effects of the drug. Our case suggests that a careful drug history should be obtained when spontaneous pneumopericardium is present in an adult without any other precipitating factors.

\section{Final diagnosis}

Pneumopericardium and pneumomediastinum due to Ecstasy and Speed.

Keywords: pneumopericardium; pneumomediastinum; Ecstasy; Speed

6 O'Neill D, Symon DN. Pneumopericardium and pneumomediastinum complicating endotracheal intubation. Postgrad Med f 1979;55:273-5.

$7 \mathrm{Hsu}$ TJ, Chen CC, Lee GW, et al. Intrapericardia spontaneous contrast echoes in pneumopericardium due to gas-forming organism. Am $\mathcal{F}$ Cardiol 1986;58:1141-4.

8 Toledo TM, Moor Jr WL, Nash DA, et al. Spontaneous pneumopericardium in acute asthma. Case report and review of the literature. Chest 1972;62:118-20. 